\title{
EVERY FINITE ABELIAN GROUP IS THE BRAUER GROUP OF A RING
}

\author{
T. J. FORD
}

\begin{abstract}
Given an arbitrary finite abelian group $\boldsymbol{G}$ a ring $\boldsymbol{R}$ is constructed using cohomological techniques from algebraic geometry whose Brauer group is $G$. If $G$ is a cyclic group, then $R$ can be taken to be a three-dimensional noetherian integral domain. If $G$ is not a cyclic group the ring $R$ is a three-dimensional noetherian ring. At the expense of raising the dimension of $R, R$ can be chosen to be a domain. We also calculate $B(R[x, 1 / x]$ for $R$ a commutative noetherian regular ring containing a field of characteristic zero.
\end{abstract}

0. Introduction. Let $A=\mathrm{C}[x, y] /\left(y^{2}-x^{2}(x+1)\right)$ be the affine coordinate ring of the nodal cubic curve $y^{2}-x^{2}(x+1)$ over the complex number field. Let $n>1$ be an integer, let $f_{n}=z y^{n-1}-x^{n}$ and let $B_{n}$ be the subring of the localized ring $\mathrm{C}[x, y, z]\left[1 / f_{n}\right]$ consisting of all fractions $g / f_{n}^{r}$ such that $g$ is a homogeneous polynomial of degree $r \cdot n, r>0$. We show that $B\left(A \otimes_{C} B_{n}\right)=Z_{n}$. Since $B_{n}$ is the affine coordinate ring of a surface we see every finite cyclic group is the Brauer group of a three-dimensional noetherian integral domain. Since every finite abelian group $G$ is of the form $\mathbf{Z}_{n_{1}} \oplus \cdots \oplus \mathbf{Z}_{n_{3}}$ we see that $G$ is the Brauer group of the three-dimensional noetherian ring $A \otimes_{\mathrm{C}}\left(B_{n_{1}} \oplus \cdots \oplus B_{n_{2}}\right)$. \&4 uses excision techniques to calculate $B(R[x, 1 / x])$ for certain rings $R$.

In this paper cohomology is always étale cohomology and a sheaf will always mean a sheaf in the étale topology. Throughout all unexplained notation and terminology is as in [10]. Tensor products and fibred products are always over the ground field $k$ which is the field of complex numbers except in $\$ 4$ where $k$ is only required to have characteristic zero.

1. A Mayer-Vietoris sequence and an excision sequence. Let

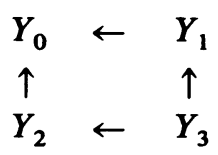

be a commutative diagram of schemes. Let $F$ be a contravariant functor from the category of schemes over $Y_{0}$ to the category of abelian groups. Suppose $F_{Y_{i}}$ (that is, $F$ restricted to $Y_{i}$ ) is a sheaf on $Y_{i}$ for each $i$. We will write $F Y_{i}$ to mean the direct image sheaf $f_{*} F_{Y_{i}}$ on $Y_{0}$ defined by $f_{*} F_{Y_{i}}(V)=F_{Y_{i}}\left(V \times Y_{Y_{0}} Y_{i}\right)$.

Received by the editors May 22, 1980 and, in revised form, October 9, 1980.

1980 Mathematics Subject Classification. Primary 13A20; Secondary 14F20. 
Definition 1.2. The diagram (1.1) is called a cartesian square with respect to the functor $F$ if the sequence $0 \rightarrow F_{Y_{0}} \rightarrow F Y_{1} \oplus F Y_{2} \rightarrow F Y_{3} \rightarrow 0$ is an exact sequence of sheaves on $Y_{0}$, where the maps are the diagonal map and the difference map, respectively.

PROPOSITION 1.3. If $(1.1)$ is a cartesian square with respect to the functor $F$ then

(a) there is a long exact sequence of cohomology:

$\cdots \rightarrow H^{n}\left(Y_{0}, F_{Y_{0}}\right) \rightarrow H^{n}\left(Y_{0}, F Y_{1}\right) \oplus H^{n}\left(Y_{0}, F Y_{2}\right) \rightarrow H^{n}\left(Y_{0}, F Y_{3}\right) \rightarrow \cdots$;

(b) if all the morphisms of (1.1) are finite morphisms, there is a long exact sequence:

$\cdots \rightarrow H^{n}\left(Y_{0}, F_{Y_{0}}\right) \rightarrow H^{n}\left(Y_{1}, F_{Y_{1}}\right) \oplus H^{n}\left(Y_{2}, F_{Y_{2}}\right) \rightarrow H^{n}\left(Y_{3}, F_{Y_{3}}\right) \rightarrow \cdots$.

Proof. (a) The proof follows from the definition of cohomology. To prove (b) we must show that $H^{n}\left(Y_{0}, F Y_{i}\right)=H^{n}\left(Y_{i}, F_{Y_{i}}\right)$. This follows from the Leray spectral sequence for each $f$ in (1.1). Since the morphisms are finite $R^{p} f_{*} F_{Y_{t}}=0$ for $p>0$ [3, Arcata, Proposition II 3.6] so the result follows.

Let $X$ be a locally noetherian regular scheme and $Z$ a closed subscheme of $X$ of codimension $d>0$. Let $F$ be a sheaf on $X$. Denote by $H_{Z}^{0}(X, F)$ the group of sections in $H_{Z}^{0}(X, F)$ with support in $Z$ and by $H_{Z}^{0}(F)$ the subsheaf of $F$ defined by the sections with support in $Z$. Define the groups (respectively, sheaves) of cohomology with support in $Z, H_{Z}^{p}(X, F)$ (respectively, $H_{Z}^{p}(F)$ ) to be the right derived functors of $H_{Z}^{0}(X, F)$ (respectively, $H_{Z}^{0}(F)$ ). The cohomology groups of $X$ and $X-Z$ are related via the long exact sequence of excision:

$$
\cdots \rightarrow H_{Z}^{n}(X, F) \rightarrow H^{n}(X, F) \rightarrow H^{n}(X-Z, F) \rightarrow H_{Z}^{n+1}(X, F) \rightarrow \cdots
$$

and the spectral sequence $H_{Z}^{p+q}(X, F) \Leftarrow H^{p}\left(Z, H_{Z}^{q}(F)\right)$. For more details on cohomology with supports in a closed subscheme see [9, V, 6 and VIII, 6.6] and [7, III, 6].

2. The Brauer group of a ruled surface. In this section a ruled surface $X$ is a nonsingular projective surface over the field $k$ together with a surjective morphism $\pi: X \rightarrow C$ to a nonsingular projective curve $C$ such that the fibre $X_{y}$ is isomorphic to $\mathbf{P}^{1}$ for every closed point $y \in C$ and such that $\pi$ admits a section. From [10, V, 2.2] we see that every ruled surface is birationally equivalent to $C \times \mathbf{P}^{1}$. Since the Brauer group is a birational invariant between complete nonsingular surfaces [7, III, Corollaire 7.2], to study the Brauer group of ruled surfaces we look at those of the form $C \times \mathbf{P}^{1}$.

Proposition 2.1. Let $C$ be a (not necessarily projective) nonsingular integral complex curve and $X=C \times \mathbf{P}^{1}$. Then $B(X)=0$.

Proof. Let $U=\operatorname{Spec} R$ be an affine open subset of $C$. Let $V=\operatorname{Spec}(k[x])$ be an open affine subset of $\mathbf{P}^{1}$ which is isomorphic to the affine line. Then $U \times V=$ $\operatorname{Spec}(R[x])$ is an open affine subset of $X$. Let $K$ be the function field of $X$. The 
natural map $B(X) \rightarrow B(K)$ is one-to-one and factors through $B(U \times V)$ [1, Theorem 7.2]. Since $C$ is a complex curve $B(R)=0$ [7, III, p. 89]; hence $B(U \times V)=$ $B(R[x])=B(R)=0[1$, Proposition 7.7]. So $B(X)=0$.

3. Every finite cyclic group is a Brauer group. Let $X$ be a noetherian integral scheme. Let $U=\operatorname{Spec} A$ be an open affine subset of $X$. Let $\tilde{A}$ be the integral closure of $A$ in its quotient field and let $\tilde{c}$ be the conductor ideal from $\tilde{A}$ to $A$, $\tilde{c}=\{r \in \tilde{A} \mid r x \in A$ for every $x \in \tilde{A}\}$. Glue together the $\tilde{U}=\operatorname{Spec} \tilde{A}$ giving the normalization $\tilde{X}$ of $X$. We define the conductor $\tilde{c}$ from $\tilde{X}$ to $X$ to be the quasicoherent sheaf of ideals $\tilde{c}$ on $\tilde{X}$. Define $c$ to be the direct image sheaf $\pi_{z} c$ under the finite morphism $\pi: \tilde{X} \rightarrow X$. For each $U=\operatorname{Spec} A$ we have $c(U)=\tilde{c}(\tilde{U})$. Let $\left(\tilde{Y}, \Theta_{\tilde{X}} / \tilde{c}\right)$ and $\left(Y, \Theta_{X} / c\right)$ be the closed subschemes of $\tilde{X}$ and $X$ corresponding to $\tilde{c}$ and $c$, respectively. There is a commutative diagram of schemes

$$
\begin{array}{lll}
\boldsymbol{X} & \leftarrow \tilde{\boldsymbol{X}} \\
\uparrow & \\
\boldsymbol{Y} & \leftarrow \tilde{\boldsymbol{Y}}
\end{array}
$$

where the vertical arrows are closed immersions, hence, are finite. We wish to see that this diagram is a cartesian square with respect to the units functor $G_{m}$. That is, we wish to see that the following is an exact sequence of sheaves on $X$ :

$$
0 \rightarrow G_{m} \rightarrow G_{m} \tilde{X} \oplus G_{m} Y \rightarrow G_{m} \tilde{Y} \rightarrow 0 .
$$

Since exactness is a local property we may assume that $X$ is affine, say $X=$ Spec $A$. Now the diagram reduces to the diagram of rings;

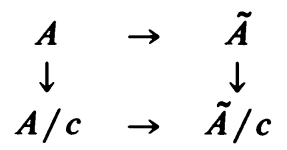

Since this diagram together with the units functor and the étale topology satisfy properties MV1 and MV2 of [2], the sequence (3.1) is locally exact, hence is exact. By Proposition 1.3 there is a long exact sequence $\cdots \rightarrow H^{n}\left(X, G_{m}\right) \rightarrow H^{n}\left(\tilde{X}, G_{m}\right)$ $\bigoplus H^{n}\left(Y, G_{m}\right) \rightarrow H^{n}\left(\tilde{Y}, G_{m}\right) \rightarrow \cdots$.

Let $C$ be a singular integral curve over $k$. Let $X$ be a noetherian integral separated regular scheme over $k$. Let $\tilde{C}$ be the normalization of $C, \tilde{Y}$ and $Y$ the closed subschemes of $\tilde{C}$ and $C$ corresponding to the conductor ideal from $\tilde{C}$ to $C$. The diagram

$$
\begin{aligned}
& C \leftarrow \tilde{C} \\
& \uparrow \\
& Y \leftarrow \tilde{Y}
\end{aligned}
$$

is a cartesian square with respect to the units functor $G_{m}$. We wish to see that

$$
\begin{array}{ccc}
C \times X & \leftarrow \tilde{C} \times X \\
\uparrow & \\
Y \times X & \leftarrow \\
Y & \tilde{Y} \times X
\end{array}
$$


is also a cartesian square with respect to the functor $G_{m}$. If $U=\operatorname{Spec} A$ is an open affine subset of $C$ and $V=\operatorname{Spec} B$ is an open affine subset of $X$ then the diagram

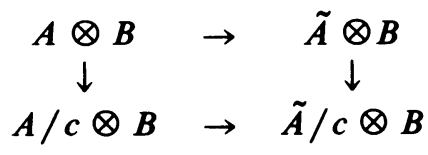

is a cartesian square since we are tensoring over the field $k$. Since the functor $G_{m}$ satisfies MV1 and MV2 of [2] for this diagram the sequence

$$
0 \rightarrow G_{m} \rightarrow G_{m}(\tilde{C} \times X) \oplus G_{m}(Y \times X) \rightarrow G_{m}(\tilde{Y} \times X) \rightarrow 0
$$

is an exact sequence of sheaves on $C \times X$. So we have the Mayer-Vietoris sequence:

$$
\begin{gathered}
\operatorname{Pic}(C \times X) \rightarrow \operatorname{Pic}(\tilde{C} \times X) \oplus \operatorname{Pic}(Y \times X) \rightarrow \operatorname{Pic}(\tilde{Y} \times X) \rightarrow H^{2}\left(C \times X, G_{m}\right) \\
\rightarrow H^{2}\left(\tilde{C} \times X, G_{m}\right) \oplus H^{2}\left(Y \times X, G_{m}\right) \rightarrow H^{2}\left(\tilde{Y} \times X, G_{m}\right) \rightarrow \cdots
\end{gathered}
$$

Now let $C$ be the affine curve given by $y^{2}=x^{2}(x+1)$. The affine coordinate ring of $C$ is $A=k[x, y] /\left(y^{2}-x^{2}(x+1)\right)$ and $\tilde{C}=\operatorname{Spec} \tilde{A}$ is isomorphic to the affine line since $\tilde{A}=k[u]$, the map $A \rightarrow \tilde{A}$ being induced by $y \mapsto u^{3}-u$ and $x \mapsto u^{2}-1$. The conductor ideal $c$ is the maximal ideal in $A$ generated by $y+x$ and $y-x, Y$ is the origin $P=(0,0), \tilde{Y}$ consists of the two closed points $Q_{1}$ and $Q_{2}$ lying over $P$ and $\tilde{c}$ is the intersection of the two maximal ideals $(u-1)$ and $(u+1)$ in $\tilde{A}$. Therefore $\tilde{Y} \times X \cong X \amalg X$ and $Y \times X \cong X$. The sequence (3.2) becomes

$$
\begin{aligned}
\operatorname{Pic}(C \times X) & \rightarrow \operatorname{Pic}(\tilde{C} \times X) \oplus \operatorname{Pic} X \stackrel{\phi}{\rightarrow} \operatorname{Pic} X \oplus \operatorname{Pic} X \rightarrow H^{2}\left(C \times X, G_{m}\right) \\
& \rightarrow H^{2}\left(\tilde{C} \times X, G_{m}\right) \oplus H^{2}\left(X, G_{m}\right) \rightarrow H^{2}\left(X, G_{m}\right) \oplus H^{2}\left(X, G_{m}\right) \rightarrow \cdots
\end{aligned}
$$

We wish to see that the image of $\phi$ is the diagonal of Pic $X \oplus$ Pic $X$. It is clear that the image of $\operatorname{Pic}(Y \times X)$ is the diagonal of Pic $X \oplus \operatorname{Pic} X$ so we check now that the image of $\phi_{1}: \operatorname{Pic}(\tilde{C} \times X) \rightarrow \operatorname{Pic} X \oplus \operatorname{Pic} X$ is the diagonal. Let $\mathcal{L}$ be an invertible sheaf on $\tilde{C} \times X$ in the Zariski topology. Let $f_{1}: Q_{1} \times X \rightarrow C \times X$ and $f_{2}: Q_{2} \times X \rightarrow C \times X$ be the closed immersions. The map $\phi_{1}$ is induced by $\mathcal{L} \mapsto$ $\left(f_{1}^{*} \mathcal{L}, f_{2}^{*} \mathcal{L}\right)$ where we are viewing sheaves on $Q_{1} \times X$ and $Q_{2} \times X$ as sheaves on $X$. By [10, Proposition II 6.6], Pic $X \cong \operatorname{Pic}(\tilde{C} \times X)$ by the map $\Re \mapsto \pi^{*} \Re$ where $\pi: \tilde{C} \times X \rightarrow X$ is the projection. Since $\pi f_{1}=1_{X}$ and $\pi f_{2}=1_{x}$ we see that $f_{1}^{*}$ and $f_{2}^{*}$ are both inverses for $\pi^{*}$. So $f_{1}^{*}=f_{2}^{*}$ and $f_{1}^{*} \mathcal{L}$ is isomorphic to $f_{2}^{*} \mathcal{L}$ as $\theta_{X}$ modules. So we see that the image of $\phi$ is the diagonal of Pic $X \oplus$ Pic $X$. The following sequence is exact:

$$
\begin{aligned}
0 & \rightarrow \operatorname{Pic} X \rightarrow H^{2}\left(C \times X, G_{m}\right) \rightarrow H^{2}\left(\tilde{C} \times X, G_{m}\right) \oplus H^{2}\left(X, G_{m}\right) \\
& \rightarrow H^{2}\left(X, G_{m}\right) \oplus H^{2}\left(X, G_{m}\right) .
\end{aligned}
$$

We will need the following.

LEMMA 3.4. Let $X$ be a regular affine surface of finite type over the field $k$. Then $H^{p}\left(X, G_{m}\right)=0$ for $p>2$. 
Proof. By [3, Arcata, Théorème IV 6.4], $H^{p}(X, F)=0$ for $p>2$ if $F$ is a torsion sheaf. So if $\mu_{n}$ denotes the kernel of the $n$th power map in the exact sequence of sheaves

$$
0 \rightarrow \mu_{n} \rightarrow G_{m} \rightarrow G_{m} \rightarrow 0,
$$

we see that $H^{p}\left(X, \mu_{n}\right)=0$ for all $p>2$ and for every $n>0$. From the long exact sequence of cohomology associated to (3.5), $H^{p}\left(X, G_{m}\right) \cong H^{p}\left(X, G_{m}\right)$ by the $n$th power map for all $n>0$ and all $p>2$. However, by [7, II, Proposition 1.4] the groups $H^{p}\left(X, G_{m}\right)$ are torsion groups for $p>2$, so $H^{p}\left(X, G_{m}\right)=0$ for $p>2$.

Let $X$ be the projective plane $\mathbf{P}^{2}$ and let $Z$ be the curve whose homogeneous equation is $f_{n}=z y^{n-1}-x^{n}=0, n \geqslant 2$. Then $Z$ is a curve of degree $n$, genus $(n-1)(n-2) / 2$. If $n>2, Z$ is singular and its desingularization is isomorphic to $\mathbf{P}^{1}$. Let $U=X-Z$. Then $U$ is a regular affine surface whose coordinate ring $B_{n}$ is the set of all homogeneous elements of degree zero in the localized ring $k[x, y, z]\left[1 / f_{n}\right]$. We will see that $\operatorname{Pic}\left(B_{n}\right)=\mathbf{Z}_{n}$ and $B\left(B_{n}\right)=0$.

To calculate the Picard group of $U$ consider the exact sequence $0 \rightarrow \mathbf{Z} \rightarrow \operatorname{Pic}(X)$ $\rightarrow \operatorname{Pic}(U) \rightarrow 0$ where the first map is induced by $1 \mapsto Z$ [10, Proposition II 6.5]. Since $\operatorname{deg}(Z)=n$ and $\operatorname{Pic} X \cong \mathbf{Z}$ by the degree map, we see that $\operatorname{Pic}(U)=\mathbf{Z}_{n}$.

Let $Y$ be the line at infinity on $X$. The exact sequence (1.4) becomes

$$
\cdots \rightarrow B(X-Y) \rightarrow H_{Y}^{3}\left(X, G_{m}\right) \rightarrow H^{3}\left(X, G_{m}\right) \rightarrow H^{3}\left(X-Y, G_{m}\right) \rightarrow \cdots
$$

where we replace $H^{2}\left(X-Y, G_{m}\right)$ by $B(X-Y)$ since $X-Y$ is a regular surface [7, II, Corollaire 2.2]. Since $X-Y$ is just the affine plane, $B(X-Y)=B(k[x, y])$ $=B(k)=0$. By Lemma $3.4, H^{3}\left(X-Y, G_{m}\right)=0$ so we have the isomorphism $H_{Y}^{3}\left(X, G_{m}\right) \cong H^{3}\left(X, G_{m}\right)$. Since $X$ is smooth over the field $k$ the couple $(X, Y)$ satisfies the theorem of purity for the Brauer group [7, III, Théorème 6.1]. Therefore, using [7, III, Corollaire 6.2] and the fact that the groups are torsion we have $H_{Y}^{3}\left(X, G_{m}\right)=H^{1}(Y, Q / Z)$. By [3, Arcata, II 2.1] we have $H^{1}(Y, Q / Z)=$ $\operatorname{Hom}\left(\Pi_{1}(Y), \mathbf{Q} / \mathbf{Z}\right)$ where $\Pi_{1}(Y)$ is the algebraic fundamental group of $Y$. For any connected scheme $S, \Pi_{1}(S)$ is the inverse $\operatorname{limit} \lim \operatorname{Gal}(T / S)$ over all étale Galois covers $T \rightarrow S$ (see [8]). Since $Y$ is isomorphic to $\mathbf{P}^{1}, \Pi_{1}(Y)=0$ [8, IX, Exemple 5.5]; hence $H^{3}\left(X, G_{m}\right)=0$.

Now consider the excision sequence

$$
0 \rightarrow B(X) \rightarrow B(U) \rightarrow H_{Z}^{3}\left(X, G_{m}\right) \rightarrow H^{3}\left(X, G_{m}\right) \rightarrow \cdots
$$

Since $X$ is a birationally ruled surface $B(X)=0$ by Proposition 2.1. Since $H^{3}\left(X, G_{m}\right)=0$ we have the isomorphism $B(U) \simeq H_{Z}^{3}\left(X, G_{m}\right)$. Again applying [7, III, Théorème 6.1 and Corollaire 6.2] we have $H_{Z}^{3}\left(X, G_{m}\right)=H^{1}(Z, Q / Z)$ and, by [3, Arcata, II 2.1], $H^{1}(Z, Q / Z)=\operatorname{Hom}\left(\Pi_{1}(Z), Q / Z\right)$. Since $Z$ is a rational curve with at most one cusp singularity, by $\left[8, \mathrm{IX}\right.$, Exemple 5.5], $\Pi_{1}(Z)=0$. Therefore, $H^{1}(Z, Q / Z)=0$; hence $B(U)=B\left(B_{n}\right)=0$. 
Replacing $X$ in the sequence (3.3) by $U=\operatorname{Spec} B_{n}$ and using the values Pic $U=$ $\mathbf{Z}_{n}, H^{2}\left(U, G_{m}\right)=B(U)=0$ we have the exact sequence

$$
0 \rightarrow \mathbf{Z}_{n} \rightarrow H^{2}\left(C \times U, G_{m}\right) \rightarrow H^{2}\left(\tilde{C} \times U, G_{m}\right) \rightarrow 0 .
$$

In his $\mathrm{Ph} . \mathrm{D}$. thesis [6, Theorem II 2], O. Gabber has proved the following result: if $X$ is an arbitrary scheme such that $X=U_{1} \cup U_{2}$ where $U_{1}$ and $U_{2}$ are affine schemes such that $U_{1} \cap U_{2}$ is affine, then $B(X)$ is isomorphic to the subgroup of torsion elements in $H^{2}\left(X, G_{m}\right)$. Taking torsion subgroups in (3.7) yields the exact sequence $0 \rightarrow \mathbf{Z}_{n} \rightarrow B(C \times U) \rightarrow B(\tilde{C} \times U) \rightarrow 0$. Since $\tilde{C}$ is isomorphic to $\operatorname{Spec}(k[u]), B(\tilde{C} \times U)=B\left(B_{n}[u]\right)=B\left(B_{n}\right)=0$. So we have the desired result: $B(C \times U)=B\left(A \otimes B_{n}\right)=\mathbf{Z}_{n}$.

REMARK 3.8. One can readily construct the Azumaya algebra which generates $B\left(A \otimes B_{n}\right)$ using results from [11].

REMARK 3.9. Using arguments analogous to the above one can show that $\operatorname{Pic}\left(B_{n_{1}} \otimes \cdots \otimes B_{n_{1}}\right)=\mathbf{Z}_{n_{1}} \oplus \cdots \oplus \mathbf{Z}_{n_{1}}$ and that $B\left(B_{n_{1}} \otimes \cdots \otimes B_{n_{2}}\right)=0$. Therefore, $\mathbf{Z}_{n_{1}} \oplus \cdots \oplus \mathbf{Z}_{n_{r}}$ is the Brauer group of the noetherian integral domain $A \otimes B_{n_{1}} \otimes \cdots \otimes B_{n_{r}}$.

4. The Brauer group of $R[x, 1 / x]$. The purpose of this section is to prove the following proposition. In [5] F. R. DeMeyer calculates $B(k[x, 1 / x])$ for a perfect field $k$. We obtain analogous results for certain coordinate rings of affine varieties.

Proposition 4.1. Let $R$ be a commutative neotherian regular ring which contains $a$ field of characteristic zero such that Spec $R$ is irreducible. Let $G$ be the Galois group of $R$. Then $B(R[x, 1 / x])=B(R) \oplus \operatorname{Hom}(G, \mathbf{Q} / \mathbf{Z})$.

Proof. We can assume $R$ is reduced (and hence integral since Spec $R$ is irreducible) by [4]. Let $X=\operatorname{Proj} R\left[x_{0}, x_{1}\right]$ and let $X_{0}=\operatorname{Spec} R\left[x_{0} / x_{1}\right], X_{1}=$ Spec $R\left[x_{1} / x_{0}\right]$ considered as open subsets of $X$. Let $Z=X-X_{1}$. Then $Z$ is isomorphic to Spec $R$ and is contained in $X_{0}$. Also $X_{0}-Z=X_{0} \cap X_{1}=$ Spec $R\left[x_{0} / x_{1}, x_{1} / x_{0}\right]$. We have two long exact sequences

$$
\begin{gathered}
\cdots \rightarrow H_{Z}^{n}\left(X, G_{m}\right) \rightarrow H^{n}\left(X, G_{m}\right) \rightarrow H^{n}\left(X_{1}, G_{m}\right) \rightarrow \cdots, \\
\cdots \rightarrow H_{Z}^{n}\left(X_{0}, G_{m}\right) \rightarrow H^{n}\left(X_{0}, G_{m}\right) \rightarrow H^{n}\left(X_{0} \cap X_{1}, G_{m}\right) \rightarrow \cdots
\end{gathered}
$$

Since $X_{0}$ is an open neighborhood of $Z$ we know that $H_{Z}^{n}\left(X, G_{m}\right)=H_{Z}^{n}\left(X_{0}, G_{m}\right)$. Since $X_{0} \cong X_{1}$ by an isomorphism sending $x_{0} / x_{1}$ to $x_{1} / x_{0}$ we have a commutative diagram for each $n$ :

$$
\begin{aligned}
H_{Z}^{n}\left(X, G_{m}\right) \rightarrow & H^{n}\left(X, G_{m}\right) \rightarrow H^{n}\left(X_{1}, G_{m}\right) \\
\searrow & \swarrow \approx \\
& H^{n}\left(X_{0}, G_{m}\right)
\end{aligned}
$$

Therefore the map $H_{Z}^{n}\left(X_{0}, G_{m}\right) \rightarrow H^{n}\left(X_{0}, G_{m}\right)$ is the zero map for each $n$. The sequence (4.3) yields the short exact sequence

$$
0 \rightarrow H^{n}\left(X_{0}, G_{m}\right) \rightarrow H^{n}\left(X_{0} \cap X_{1}, G_{m}\right) \rightarrow H_{Z}^{n+1}\left(X_{0}, G_{m}\right) \rightarrow 0
$$

for each $n$. For $n=2$ we have the exact sequence

$$
0 \rightarrow H^{2}\left(X_{0}, G_{m}\right) \rightarrow H^{2}\left(X_{0} \cap X_{1}, G_{m}\right) \rightarrow H_{Z}^{3}\left(X_{0}, G_{m}\right) \rightarrow 0 .
$$


By [7, II, Proposition 1.4] the $\boldsymbol{H}^{2}$ groups are torsion groups and by Gabber's result [6, Theorem II 2] we have the exact sequence

$$
0 \rightarrow B(R[x]) \rightarrow B(R[x, 1 / x]) \rightarrow H_{z}^{3}\left(X_{0}, G_{m}\right) \rightarrow 0 .
$$

Since $X$ is regular over a perfect field $k$, it is smooth over $k$ so we can replace $H_{Z}^{3}\left(X_{0}, G_{m}\right)$ with $\operatorname{Hom}(G, Q / Z)$ by [7, III, Corollaire (6.2)]. Hence the sequence becomes $0 \rightarrow B(R[x]) \rightarrow B(R[x, 1 / x]) \rightarrow \operatorname{Hom}(G, \mathbf{Q} / Z) \rightarrow 0$. Since $R$ is regular of characteristic zero, $B(R[x])=B(R)$ [1, Proposition 7.7] and the map $B(R) \rightarrow$ $B(R[x, 1 / x])$ splits; hence

$$
B(R[x, 1 / x])=B(R) \oplus \operatorname{Hom}(G, \mathbf{Q} / \mathbf{Z}) .
$$

REMARK 4.5. In [12] the Brauer group of $k\left[x_{1}, \ldots, x_{d}, x_{1}^{-1}, \ldots, x_{d}^{-1}\right]$ is calculated to be $(\mathbf{Q} / \mathbf{Z})^{n}$ where $n=d(d-1) / 2$ and $k$ is an algebraically closed field with characteristic zero. Applying Proposition 4.1 inductively and using the fact that $H^{1}\left(k\left[x_{1}, \ldots, x_{d}, x_{1}^{-1}, \ldots, x_{d}^{-1}\right], \mathbf{Q} / \mathbf{Z}\right)=(\mathbf{Q} / \mathbf{Z})^{d}$ we obtain a more concise proof of this result.

\section{REFERENCES}

1. M. Auslander and O. Goldman, The Brauer group of a commutative ring, Trans. Amer. Math. Soc. 97 (1960), 367-409.

2. L. N. Childs, Mayer-Vietoris sequences and Brauer groups of nonnormal domains, Trans. Amer. Math. Soc. 196 (1974), 51-67.

3. P. Deligne et al., SGA4 $\frac{1}{2}$, Cohomology étale, Lecture Notes in Math., vol. 569, Springer-Verlag, Berlin and New York, 1977.

4. F. R. DeMeyer, The Brauer group of a ring modulo an ideal, Rocky Mountain J. Math. 6 (1976), 191-198.

5. The Brauer group of affine curves, Brauer Groups (Evanston 1975), Lecture Notes in Math., vol 549, Springer-Verlag, Berlin, 1976, pp. 16-24.

6. O. Gabber, Some theorems on Azumaya algebras, Ph.D. thesis, Harvard University, 1978.

7. A. Grothendieck, Le groupe de Brauer. I, II, III, Dix Exposés sur la Cohomologie des Schémas, North-Holland, Amsterdam; Masson, Paris, 1968, pp. 46-188.

8. SGA1, Revêtements étales et groupe fondamental, Lecture Notes in Math., vol. 224, Springer-Verlag, Berlin and New York, 1971.

9. A. Grothendieck, M. Artin and J. L. Verdier, SGA4, Théorie des topos et cohomologie étale des schémas, Lecture Notes in Math., vol. 269, 270, 305, Springer-Verlag, Berlin and New York, 1972-1973.

10. R. Hartshorne, Algebraic geometry, Springer-Verlag, New York, 1977.

11. M. A. Knus and M. Ojanguran, A Mayer-Vietoris sequence for the Brauer group, J. Pure Appl. Algebra 5 (1974), 345-360.

12. A. R. Magid, Brauer groups of linear algebraic groups with characters, Proc. Amer. Math. Soc. 71 (1978), 164-168.

Department of Mathematics, Colorado State University, Fort Collins, Colorado 80523 